EPSC Abstracts

Vol. 15, EPSC2021-71, 2021, updated on 04 Nov 2021

https://doi.org/10.5194/epsc2021-71

Europlanet Science Congress 2021

(C) Author(s) 2021. This work is distributed under

the Creative Commons Attribution 4.0 License.

\title{
The ESA Hera mission to the binary asteroid (65803) Didymos: Planetary Defense and Science
}

\author{
Patrick Michel ${ }^{1}$, Michael Kueppers ${ }^{2}$, Alan Fitzsimmons ${ }^{3}$, Simon Green ${ }^{4}$, Monica Lazzarin ${ }^{5}$, Stephan \\ Ulamec $^{6}$, Ian Carnelli ${ }^{7}$, Paolo Martino ${ }^{7}$, and the Hera Science Team ${ }^{*}$ \\ ${ }^{1}$ Université Côte d'Azur, Observatoire de la Côte d'Azur, CNRS, Laboratoire Lagrange, Nice, France (michelp@oca.eu) \\ ${ }^{2}$ ESA-ESAC, Camino bajo del Castillo S/N, Urbanización Villafranca del Castillo, 28692 Villanueva de la Cañada, Madrid, Spain \\ ${ }^{3}$ Astrophysics Research Centre, Queen's University Belfast, Belfast BT7 1NN, Northern Ireland \\ ${ }^{4}$ School of Physical Sciences, The Open University, Robert Hooke Building, Walton Hall, Milton Keynes MK7 6AA, UK \\ ${ }^{5}$ Department of Physics and Astronomy, Padova University, Vicolo dell'Osservatorio 3, 35122 Padova, Italy \\ ${ }^{6}$ DLR RB-MUSC, Linder Höhe 1, 51147 Cologne, Germany \\ ${ }^{7}$ ESA-ESTEC, Keplerlaan 1, Noordwijk 2200 AG, The Netherlands \\ *A full list of authors appears at the end of the abstract
}

The Hera mission is in development for launch in 2024 within the ESA Space Safety Program. Hera will contribute to the first deflection test of an asteroid, in the framework of the international NASAand ESA-supported Asteroid Impact and Deflection Assessment (AIDA) collaboration. Hera will also offer a great science return.

\section{Introduction}

The impact of the NASA DART spacecraft on the $160 \mathrm{~m}$-diameter natural satellite called Dimorphos of the binary asteroid 65803 Didymos in late September 2022 will change its orbital period around Didymos. As Didymos is an eclipsing binary, and close to the Earth on this date, the change can be detected by Earth-based observers. Before impact, DART will deploy the Italian LICIACube that will provide images of the first instants after impact. ESA's Hera spacecraft will rendezvous Didymos four years after the impact. It will perform the measurements necessary to understand the effect of the DART impact on Dimorphos, in particular its mass, its internal structure, the direct determination of the momentum transfer and the detailed characterization of the crater left by DART.

\section{Planetary Defense return}

Hera will characterize in details the properties of a Near-Earth Asteroid that are fully relevant to planetary defense. Its objectives related to the deflection demonstration are the following:

-Measuring the mass of Dimorphos to determine the momentum transfer efficiency from DART impact.

-Investigating in detail the crater produced by DART to improve our understanding of the cratering process and the mechanisms by which the crater formation drives the momentum transfer efficiency.

- Observing subtle dynamical effects (e.g. libration imposed by the impact, orbital and spin excitation of Dimorphos) that are difficult to detect for remote observers.

- Characterising the surface and interior of Dimorphos to allow scaling of the momentum transfer efficiency to different asteroids. 


\section{Science return}

Even if its requirements are driven by planetary defense, Hera will also provide unique information on many current issues in asteroid science. The reason is that our knowledge of these fascinating objects is still poor, especially for the smallest ones. The recent data obtained by the JAXA Hayabusa 2 and NASA OSIRIS-REx missions have revolutionized our understanding of carbonaceoustype Near-Earth Objects. Hera has the the potential to do similar as it will rendezvous for the first time with a binary asteroid. Its secondary has a diameter of only $160 \mathrm{~m}$ in diameter. So far, no mission has visited such a small asteroid. Moreover, for the first time, internal and subsurface properties will be directly measured. From small asteroid internal and surface structures, through rubble-pile evolution, impact cratering physics, to the long-term effects of space weathering in the inner Solar System, Hera will have a major impact on many fields. How do binaries form? What does a $160 \mathrm{~m}$-size rock in space look like? What is the surface composition? What are its internal properties? What are the surface structure and regolith mobility on both Didymos and Dimorphos? And what will be the size and the morphology of the crater left by DART, which will provide the first impact experiment at full asteroid scale using an impact speed close to the average speed between asteroids? These questions and many others will be addressed by Hera as a natural outcome of its investigations focused on planetary defense.

\section{Instruments}

Hera is equipped with the following payload:

- The Asteroid Framing Cameras are both science and navigation cameras. They will provide the target global properties as well as local geomorphology and will investigate the crater. They will also measure the mass of Dimorphos through the "wobble" motion of Didymos.

- The Planetary ALTimeter (PALT) will measure the distance to the target and, from close distance, derive shape and topography information complementary to the shape information in framing camera images.

- A thermal infrared imager (TIRI) will provide information about thermal properties and spectral information in the mid-infrared.

- The Hyperscout-H hyperspectral imager will provide mineralogical information in the spectral range between 450 and $950 \mathrm{~nm}$.

- Milani is a 6 unit cubesat that will carry the ASPECT Fabry-Perot imager to derive mineralogical information, and a thermogravimeter for measuring the abundance and constraining the composition of ambient dust particles.

- Juventas is a 6 unit cubesat that will carry a monostatic low-frequency radar, and a gravimeter to derive interior and surface properties of the asteroids. Its landing on Dimorphos will also allow an estimate of the surface response to a very slow impact.

- The radioscience experiment will measure the gravity field of the Didymos system. It will work in two ways: measurements of the acceleration of the Hera spacecraft by the asteroid pair through the radio link between earth and Hera will be used as well as the intersatellite link between Hera and the two cubesat, which will measure the gravitational parameters from the relative position and velocity of the three spacecraft.

\section{NEO-MAPP}

NEO-MAPP (Near Earth Object Modelling and Payload for Protection) is a project funded by the H2020 program of the European Commission. Hera is its reference mission, and most of the NEOMAPP activities are aimed at supporting the preparation of Hera. The main goal of NEO-MAPP is to provide significant advances in our modeling of impact physics, binary dynamics and internal properties, as well as in instrumentations and associated measurements by a spacecraft (including those necessary for the physical and dynamical characterization in general). In particular, innovative and synergetic measurement and data-analysis strategies are developed that combine multiple payloads, to ensure optimal data exploitation for Hera and other NEO missions.

\section{Conclusion}

The measurements performed by Hera will thus provide unique information on many current issues in asteroid science and therefore, the scientific legacy of the Hera mission will extend far beyond the core aims of planetary defense. Hera is thus an amazing European contribution to the international planetary defense and asteroid exploration era. 


\section{Acknowledgements}

We thank ESA and CNES for support. We also acknowledge funding from the European Union's Horizon 2020 research and innovation programme under grant agreement No 870377 (project NEOMAPP).

Hera Science Team: Patrick Michel, Adriano Campo Bagatin, Benoît Carry, Sébastien Charnoz, Julia de Leon, Alan Fitzsimmons, Simon F. Green, Alain Hérique, Martin Juzi, Özgür Karatekin, Tomas Kohout, Monica Lazzarin, Naomi Murdoch, Tatsuki Okada, Ernesto Palomba, Petr Pravec, Colin Snodgrass, Paolo Tortora, Kleomenis Tisganis, Stephan Ulamec, Jean-Baptiste Vincent, Kai Wünnemann, Elisabetta Dotto, Nancy Chabot, Andy F. Cheng, Andy Rivkin, Olivier Barnouin, Carolyn Ernst, Angela Stickle, Derek C. Richardson, Cristina Thomas, Masahiko Arakawa, Hirdy Miyamoto, Akiko Nakamura, Seiji Sugita, Makoto Yoshikawa, Paul Abell, Erik Asphaug, Ronald-Louis Ballouz, William F. Bottke Jr., Dante S. Lauretta, Kevin J. Walsh 